\title{
An Overview Of How Microscopy Is Employed By the Oregon State Police Forensic Services Division.
}

\author{
Celeste M. Grover ${ }^{1}$ \\ ${ }^{1}$ Oregon State Police Forensic Services Division, Clackamas, Oregon
}

Microscopy is used in some capacity in many of the forensic science disciplines found within a typical full service forensic science laboratory system. The information gathered from the observations made possible by microscopes is critical in the development of conclusions made about evidence in a crime. The following is a general overview of how microscopy is employed in some of the forensic disciplines of the Oregon State Police Forensic Services Division.

The low magnification stereoscope can be utilized in any section examining physical evidence. It can aid in the ability for the forensic scientist to see potential trace and/or biological evidence not visible to the unaided eye.

In the biology section compound microscopes are used to determine if spermatozoa are present on evidence typically related to cases involving an alleged sexual assault. An extract of suspected material is heat fixed to a slide and stained with Christmas Tree stain (i.e., nuclear fast red/picroindigocarmine). The slide is then examined for the presence of spermatozoa via bright field, phase contrast, and/or oil immersion. Confirmation of any sperm is done at 400X magnification or greater. (Figure 1)

Both stereomicroscopy and polarized light microscopy are utilized by forensic scientists in the controlled substances section. Observations of plant morphology by stereomicroscopy are crucial to the identification of marijuana. Suspected plant material is examined for the presence of a combination of identifying features including: cystolith hairs, cover hairs, styles/stigmas, glandular hairs, serrated leaves, and mottled seeds. Low magnification stereomicroscopes can also aid in the determination of worn pharmaceutical and clandestine tablet imprints. Additionally, polarized light microscopy (PLM) can be used to observe microcrystalline tests specific for certain drugs or other compounds of interest. (Figure 2)

The stereomicroscope plays a key role in the firearms and toolmarks section of the laboratory. In order to determine if bullets or cartridge cases could have been fired from a particular firearm, the microscopic marks imparted by the barrel or firing pin are examined and compared to a bullet or cartridge case known to have been fired from the firearm in question (test fire). The questioned bullet/cartridge case is compared side by side with the test fire using a comparison microscope (two stereomicroscopes attached with an optical bridge) at 6-70x magnification. Toolmark comparisons are done in a similar fashion comparing marks in question to a particular implement. Additionally, stereomicroscopes are used to aid in observation and examination of gun powder particles and obliterated serial numbers. (Figure 3)

Microscopy is a cornerstone in the examination of trace evidence. Trace evidence comes in many forms, and as such a variety of microscopes and microanalytical techniques are used. The initial exam of most items of evidence for trace evidence involves a screening step utilizing the stereomicroscope (5120x). Targeted particles can include: glass, paint, hairs, fibers, low explosive residues, or other miscellaneous debris of interest. Once collected, the particles may then be examined further using 
additional microscope-influenced techniques at higher magnification (50-400x) such as PLM, Fourier Transform Infrared microspectroscopy, microspectrophotometry (MSP), fluorescence microscopy, microcrystalline tests, and refractive index measurement. When applicable, the morphological characteristics and instrumental data of the unknown are compared to a known standard. In the case of hair and fiber evidence, the microscopic characteristics of the unknown sample can be compared side by side to those of a sample from a known source using a compound comparison microscope. (Figure 4)
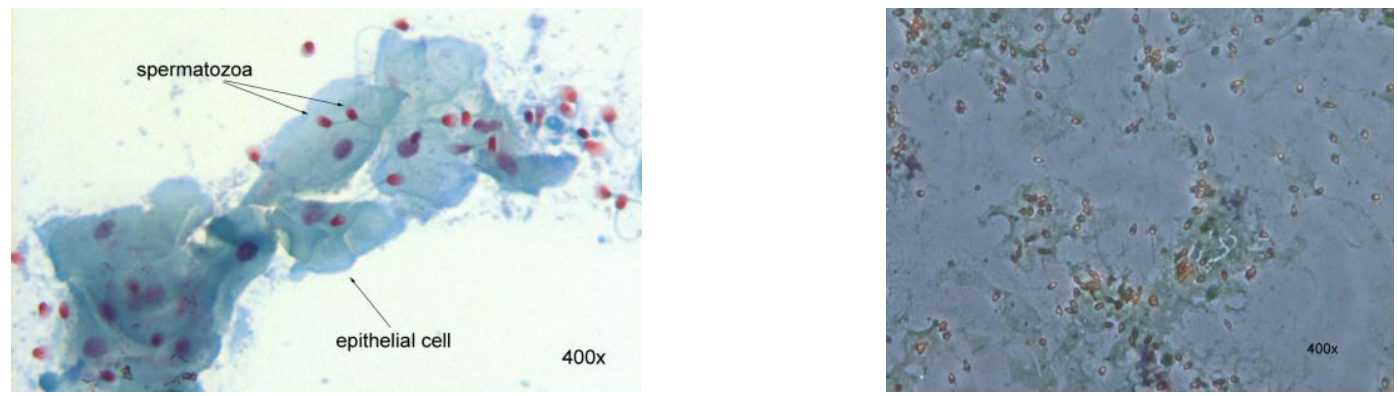

Figure 1. Christmas Tree stain in: brightfeld (left); phase contrast (right)
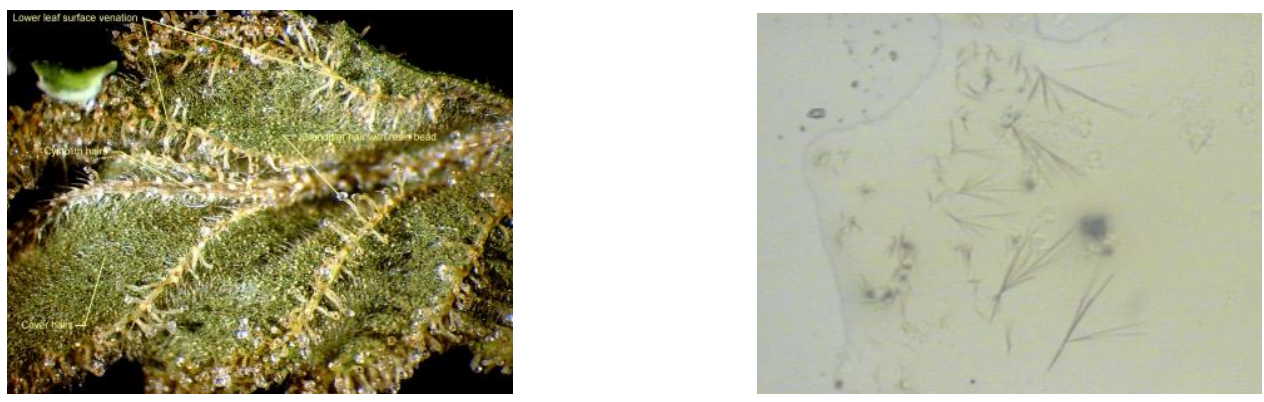

Figure 2. marijuana leaf (left); gold bromide microcrystalline test for heroin (right)

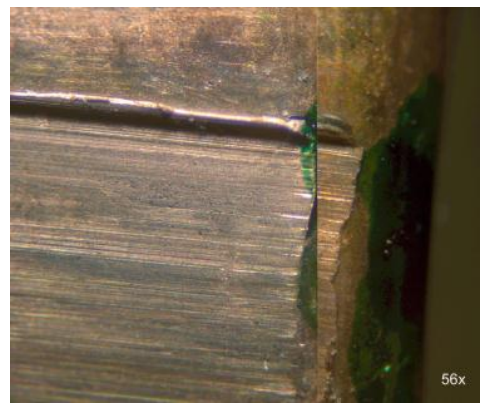

Figure 3. bullet comparison (left); shot pellet in bone (right)


Figure 4. camel hair (left); paint chip cross section (center); silver nitrate microcrystalline test for potassium sulfate (right) 